\title{
Sustainability of health benefits: Challenges faced by councils health management teams in sustaining comprehensive emergency care services after project phase out. The case of Rufiji, Kilombero and Ulanga districts
}

Josephine Shabani * Iddagiovana Kinyonge, Hadija Kweka, Selemani Mbuyita, Ahmed Makemba, Godfrey Mbaruku Ifakara Health Institute, Dar es Salaam, Tanzania

Received: January 13, 2015

DOI: $10.5430 /$ jha.v4n4p1
Accepted: April 3, 2015

Online Published: April 28, 2015

\begin{abstract}
Background: Attention to the sustainability of health intervention programs is increasing not only in developing countries but also in developed countries together with international development agencies. However, consensus on operational definitions of sustainability and determinants of sustainability is still at an early stage. While much progress has been made in the development of successful interventions to promote health, too few interventions achieve long term sustainability. Implementation of EMPOWER project in collaboration with World Lung Foundation (WLF) have increased accessibility of comprehensive emergency obstetric care (CEmOC) by upgrading health centers which were formerly not providing CEmOC services in the three rural districts in Tanzania. Although the WHO standards of CEmOC coverage in the project districts was above the requirement, but accessing these health facilities which provides CEmOC was so difficult due to various factors like geographical (mountains, rivers, seasonal roads), locations of these health facilities (like in one district the it was located at a corner of the district), unreliable referral system and poor functionality of these health facilities especially in terms of emergency preparedness etc. all these factors lead to less/poor accessibility to CEmOC. The upgraded facilities include Kibiti in Rufiji district, Mlimba in Kilombero district, Mwaya and Mtimbira in Ulanga district. Objective: To explore challenges of sustaining upgraded health centers and impact on service utilization after project phase out among rural communities in Tanzania.

Methods: Purposeful criterion-based selection of the upgraded health centers (those providing CEmOC) was used in the three districts two years after project phase-out. Secondary data analysis of the quantitative data which was collected during and after the project was done. The following services were assessed; total number of facility deliveries, average number of cesarean section (CS), ante natal care (ANC) attendance, post natal care (PNC) attendance, family planning (FP) use and partograph to monitor the progress of labor. Qualitative data involved key informant interviews of council health management teams (CHMT) and facility in charges.Monitoring data, evaluation and observation of various $\mathrm{CEmOC}$ and $\mathrm{MNCH}$ related indicators were also done. Four upgraded health centers (Mwaya, Mtimbira, Mlimba and Kibiti) were used as case studies to generate learning reported in this paper.

Results: Two years post project, the utilization of most of the services like number of deliveries and CS performed better and were maintained in upgraded health centers which receive regular assistance (Mwaya and Mlimba) than Kibiti health center which received minimal support. Health workers remained committed to sustain the practices promoted in the interventions
\end{abstract}

*Correspondence: Josephine Shabani; Email: jshabani@ihi.or.tz; Address: Ifakara Health Institute, Po Box 78373, Dar es Salaam, Tanzania. 
despite of the noted challenges.

Conclusions: Benefits of introduced health innovations such as upgrading of health centers for CEmOC can only be sustained if a sustainability strategies are integrated at early stages of project design and carried forward in routine district health planning processes.

Key Words: Sustainability, Upgraded health centers

\section{INTRODUCTION}

Annually, it is estimated that 536,000 women die worldwide from pregnancy and childbirth related conditions, as do 11 million under-fives, of which 4.4 million are newborns, most of these deaths occur in Sub Saharan Africa. ${ }^{[1]}$ According to the 2012 National Population and Housing Census, Tanzania Mainland is populated with 44,928,923, out of which $75 \%$ live in rural areas. The annual growth rate is $2.7 \%$ with life expectancy at birth being 54 years for males and 56 years for females. The current total fertility rate in Tanzania stands at 5.4 , which is high. There are regional variations with urban rural disparities, where rural women have higher fertility rates than their urban counterparts. ${ }^{[2]}$

The Maternal Mortality Ratio in Tanzania has remained high for the last 10 years with no decline, the current level is estimated at 454 per 100,000 live births while neonatal deaths is estimated at 26 per 1,000 live births, this accounts for $47 \%$ of the infant mortality rate which is estimated at 51 per 1,000 live births. ${ }^{[2]}$

In Tanzania, the high fertility rate combined with the low contraceptive prevalence rate increase the lifetime risk of maternal death. Unfortunately, for the majority of women, especially the poor and disadvantaged groups, the pathway to safe motherhood are blocked by the underlying factors that lead to delays in accessing appropriate care. These include: delays at individual, household and community levels in making the decision to seek appropriate care; delay in reaching the appropriate facility due to financial and geographical obstacles (transport); and delay at the facility in receiving appropriate care due to a weak health system and poor quality of care. ${ }^{[3,4]}$

\section{Initiatives to improve maternal and child health}

In 1989, Tanzania adopted the Safe Motherhood Initiative (SMI), following the official launch of the Global Safe Motherhood Initiative in 1987 in Nairobi, Kenya. However, maternal and perinatal mortality rates have remained unacceptably high in the country, as is the case in other African countries. Subsequently, the 1994 International Conference for Population and Development, and Millennium Development Goals of 2000, highlighted the need to re-focus the strategies for reducing the persistently high maternal and new-born mortal- ity by setting clear targets for improving maternal and child health. ${ }^{[3]}$

In Tanzania, specific attempts have been made to address maternal and newborn health challenges through the National Health Policy, Health Sector Reforms and the Health Sector Strategic Plan IV (2009-2015). ${ }^{[5]}$ In addition to this, the Reproductive and Child Health Strategy (2010-2015) has also been formulated to respond to the problem. Improving maternal and child health is also a major priority area in the National Strategy for Growth and Reduction of Poverty (MKUKUTA) 2005-2010. One of the goals clearly outlined in the second cluster of the strategy is to improve survival, health and wellbeing of all children and women, as well as other vulnerable groups. Under this goal, MKUKUTA sets an operational target related to maternal and child health, which is crucial in monitoring progress towards reducing morbidity and mortality among pregnant women, young girls and newborns.

Although much progress has been made in the development of successful interventions to promote health, too few interventions achieve long term sustainability. As a result sustainability has remained as one of the key challenges in public health. ${ }^{[6]}$ According to the International Fund for Agricultural Development (IFAD) report of 2009, sustainability is defined as ensuring that the institutions supported through projects and the benefits realized are maintained and continue after the end of the project. The US Agency for International Development in 1988 also defined a sustainable project as the one which is able to deliver an appropriate level of benefits for an extended period of time after major financial, managerial and technical assistance from an external donor is terminated. Most of the innovations that are initially successful, fail to become part of the habits and routines of the host organizations and communities, the major challenge being the high dependency of donor funding to sustain the program. ${ }^{[7]}$ Over the years scientists have been struggling in fostering the sustainability of interventions in community systems because they want to see the possibility of maintaining or increasing effects achieved during a research phase. Sustainability of health interventions is critical because it is more than continuation of interventions since in 
the long run it should result into the exchange of knowledge and resources. ${ }^{[8]}$ In addition, the health providers who would have gained "added skills" will be expected to provide better services on a long term basis.

Meanwhile health facility related interventions have generated excitement, innovation, and exploration of alternative approaches over the last two decades, there has been tensions that health researchers face about transferring innovations to health systems, changing program delivery from intervention context controlled by researchers to program delivery controlled by health facilities and community organizations, as well as sustaining long term effects of interventions. ${ }^{[9]}$

Emergency Obstetric Care (EmOC) refers to the care needed to handle potentially life-threatening direct and indirect obstetric complications that affect an estimated $15 \%$ of women during pregnancy, at delivery or in the postpartum period. The provision of EmOC is assessed by using EmOC process indicators recommended by the United Nations. ${ }^{[9]}$ These indicators measure activities that lead to the desired goal of reduction in maternal deaths. The indicators are based on the fact that, certain types of obstetric services must be available in sufficient quantity and used by women who need them at that particular time.

There are eight signal functions, of which six can be performed at the level of a health center or dispensary. A facility performing all six signal functions is categorized as a Basic EmOC facility (BEmOC). Two additional functions are often available only at a hospital; a facility performing all eight functions qualifies as a Comprehensive EmOC facility (CEmOC). The BEmOC signal functions are to: administer parenteral (intravenous or by injection) antibiotics, administer parenteral oxytocic drugs, administer parenteral anticonvulsants, perform manual removal of placenta, perform removal of retained products, and perform assisted vaginal delivery (vacuum extraction, forceps delivery). The CEmOC functions are all six Basic Signal Functions plus to: perform surgery (cesarean section [CS]) and blood transfusion. It should be noted in order to be able to perform surgery, anesthesia is required. ${ }^{[10,11]}$ This paper uses the case of upgraded health centers providing CEmOC services to build a case on sustainability of several $\mathrm{MNCH}$ related services.

\section{Material AND Methods}

\subsection{Intervention}

EMPOWER Project was developed following the phasing out of Tanzania Essential Health Interventions Project (TEHIP). TEHIP lessons indicated that advancing the evidence based interventions are only possible if the health system is strengthened. In 2007/2008 Ifakara Health Insti- tute (IHI) launched EMPOWER project which implemented a systems wide approach to address Maternal, Newborn and Child Health morbidity and mortality in Kigoma, Rufiji, Kilombero and Ulanga District Councils . They major aim of the project was to increase access both basic and CEmOC. This was done through skills development of health providers, capital investment in terms of infrastructure development and supply of equipment for health centers that were upgraded to provide comprehensive emergency care. The selection of facility to upgrade was done by the district in consideration of distance from district hospital and influx of patients. Although by WHO standers of CEmOC coverage in the three districts is above the requirement, but accessing these health facilities is so difficult in these districts due to various factors. Some of the factors are geographical (mountains, rivers, seasonal roads), locations of these health facilities in a particular district (located at a corner of the district), unreliable referral system and neither local transport, far away from their place (need of relative for help/place to stay), poor functionality of these health facilities especially in terms of emergency preparedness, few health workers and constant unavailability of equipment and supplies, all these factors lead to less/poor accessibility to CEmOC. As a result total of four health centers were upgraded, one health center (Mlimba) was upgraded in Kilombero district. Mlimba health center is located $150 \mathrm{~km}$ from Saint Francis Designated District Hospital (SFDDH). Two health centers were upgraded in Ulanga district (Mtimbira and Mwaya). Mtimbira and Mwaya are $100 \mathrm{~km}$ and $40 \mathrm{~km}$ from Mahenge district hospital respectively. The fourth health center was Kibiti health center which is $84 \mathrm{~km}$ away from Utete district hospital. Upgrading of the most remote health centers to provide CEmOC services was intended to reduce phase two delays (delays to reach care). These interventions were supported by Comic Relief (UK), World Lung Foundation (WLF) as well as the Ministry of Health and Social Welfare (MOHSW), Tanzania .

\subsection{Study approach and strategy}

Purposeful criterion-based selection of the upgraded health centers (those providing CEmOC) was used in the three project districts two years after project phase-out. The health center were selected based on following criteria: (i) The health center is upgraded; (ii) It is not in any donor funding for a year; and (iii) The health center continues to provide CEmOC.

\subsection{Data collection and analysis}

The assessment took two weeks and two research teams were used where by both qualitative and quantitative dataset were collected. Quantitative data was collected from registers in order to assess various $\mathrm{MNCH}$ indicators for compari- 
son of service utilization attained when the upgraded health centers were receiving support from the project and after the termination of project support. The following services were assessed; total number of facility deliveries, average number of $\mathrm{CS}$, ante natal care (ANC) attendance, post natal care (PNC) attendance, family planning (FP) use and partograph to monitor the progress of labor. Monitoring data, documentary review and observation of various $\mathrm{CEmOC}$ and $\mathrm{MNCH}$ related indicators were also done. The upgraded health centres were used as case studies to generate learning reported in this paper. The qualitative interviews involved key informant interview technique where by the guiding questionnaire was administered to council health management teams (CHMTs), health canters in charges, health providers and clients respectively. All these are either direct or indirect clients of the upgraded health centers in the three project districts. Semi structured interviews and key informants interviews were used as they allow respondents to open up and express themselves in their own terms and experience on the current situation of the health centers after being upgraded. This information was gathered to explore experiences and recommendations of respondents on sustaining $\mathrm{CEmOC} \mathrm{ac-}$ tivities after the project funding ended. The quantitative data was cleaned, processed and analysed using Stata 12 software (College Station, Texas 77845 USA). Qualitative data were evaluated in five major themes; (i) Leadership continuity; (ii) Planning capacity; (iii) Skills management; (iv) Continuity of CEmOC services and capacity to deliver CEmOC; (v) Sustainability challenges.

\section{RESULTS}

\subsection{During project implementation}

\section{Increase in service utilization}

Following introduction of CEmOC services (June 2010) the trends of total deliveries increased remarkably in all four upgraded health centers i.e. Kibiti, Mlimba, Mtimbira and Mwaya (see Figure 1). On average, monthly deliveries increased by as much as $300 \%$ at Mlimba and Kibiti upgraded health centers, whereas Mtimbira and Mwaya upgraded health centers the increase ranged from $100 \%$ to $200 \%$.

There was also a notable increase in the average number of CS cases managed in these obstetric health centers (see Figure 2). During this period the health facilities recorded zero maternal deaths during or after the CS for all upgraded health centers this signifies the quality and safety of the procedure. After introduction of CS services the number of referrals decreased significantly because most of obstetric complications could be handled at the upgraded health center. Except for Kibiti upgraded health center which showed a decreasing trend (see Figure 2), the rest of health centers performed well in this indicator.

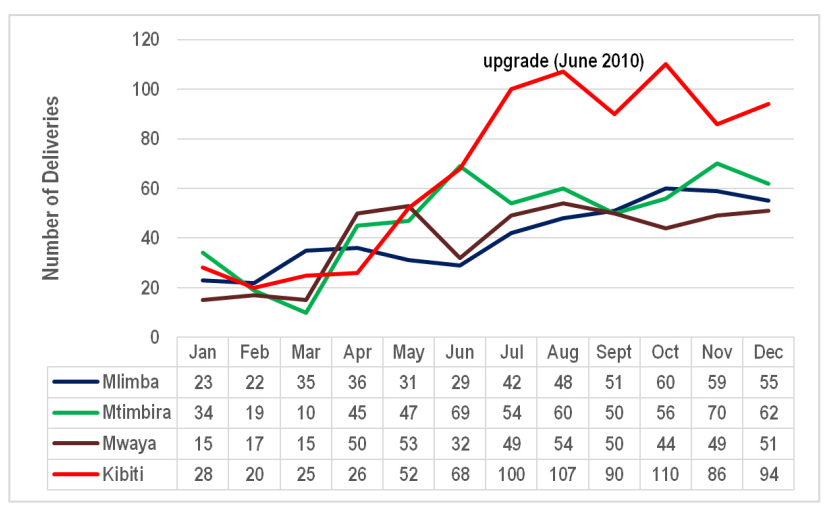

Figure 1. Trend in the monthly deliveries before and after launching CEmOC services (June 2010) in four remote health centers in Tanzania

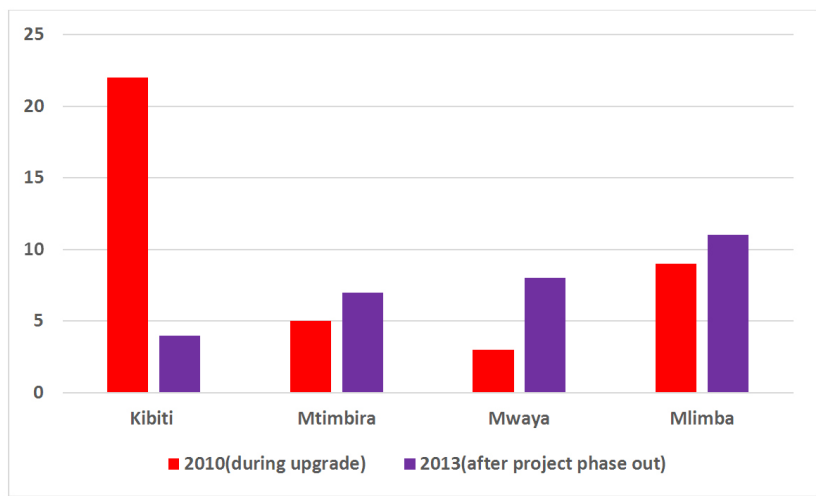

Figure 2. Average number of CS per year for upgraded health centers

Upgrading of health centers also stimulated other services like $\mathrm{ANC}$ total attendance, $\mathrm{ANC} \geq 4$ visits, $1^{\text {st }} \mathrm{ANC}$ visit at $<20$ weeks (see Figure 3 ) as well as PNC at least 1 visit, FP new cases, and partograph use (see Figure 4). Generally there is an average moderate performance of indicators with the results varying across the four upgraded health facilities as shown by Figures 3 and 4 .

\subsection{After project phase out}

\section{Decrease in service utilisation}

Two years after project phase out in 2013, monthly deliveries fluctuated particularly at the Kibiti upgraded health center. The medical officer in charge of the Kibiti upgraded health center reported that this facility had frequently stock outs of drugs and supplies, so the clients tended to leave whenever these were out of stock. This situation can further be seen in Figure 5 after project phase in 2013, monthly deliveries fluctuated, particularly at the Kibiti upgraded health center. 
However, for other upgraded centers (Mlimba, Mwaya and Mtimbira) the situation is much better because they normally get some assistance on essential medicines and supplies. The same condition is observed for the average number of CS after project phase out (see Figure 2).

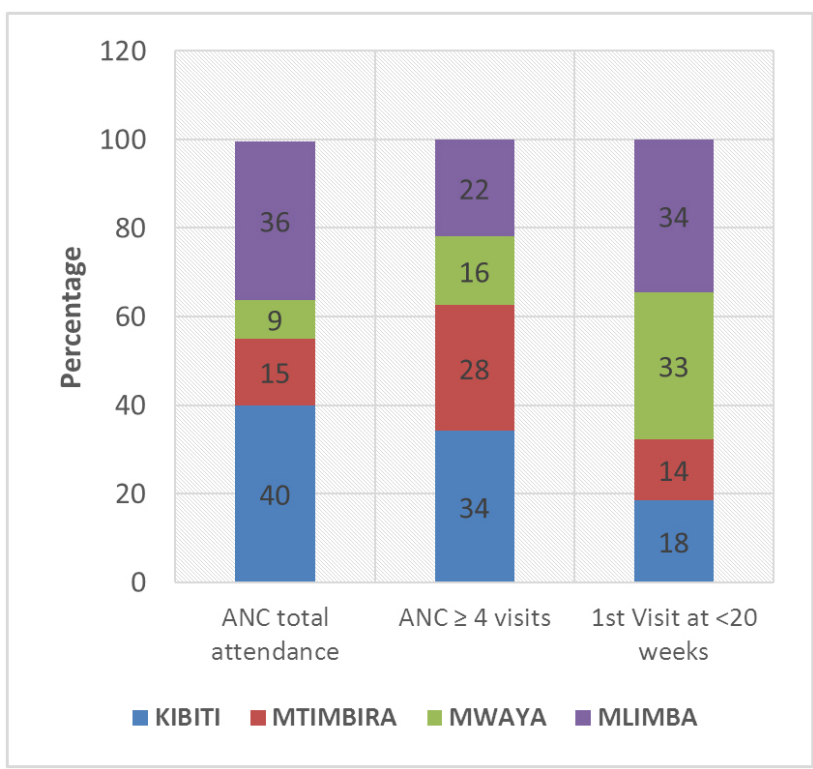

Figure 3. Distribution of $A N C$ attendance $A N C \geq 4$ visits and $1^{\text {st }}$ visits at $<20$ weeks for upgraded health centers

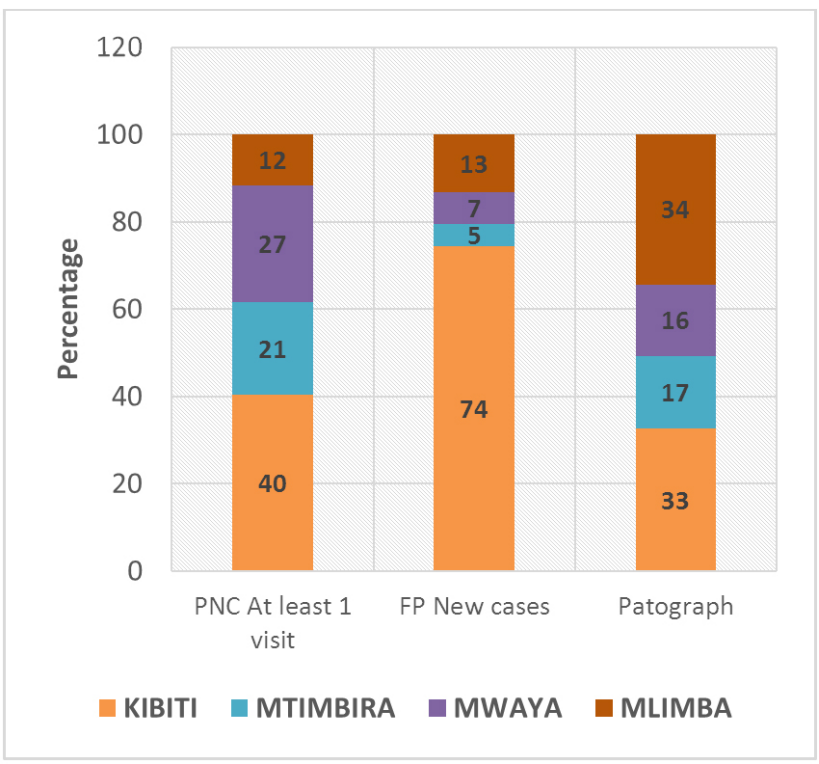

Figure 4. Distribution PNC at least 1 visit, FP new case and the use of partographs for upgraded health centers

\subsection{Functionality of CHMTs}

\subsubsection{Leadership continuity}

The results showed that at the district levels the leaders i.e. CHMT and district medical officers have changed a lot during and even after project implementation. This might have an impact on the programs and projects like EMPOWER which is doing implementation at district level. This is because the new leader has to orient him/herself with the project which normally has timelines.

\subsubsection{Planning capacity}

The study also realized that $60 \%(\mathrm{n}=18)$ of CHMT members who were interviewed have received planning training (PlanRep) done by EMPOWER project 2-3 times. But the results showed that most of those who received training have been transferred to other places and those trained are not giving feedback.

\subsubsection{Skills management}

In the upgraded health centres visited, most of the guidelines, job aids and posters were there and have been used by health providers including facility incharges, but the challenge is sometimes they are misplaced because more than one health provider is using them.

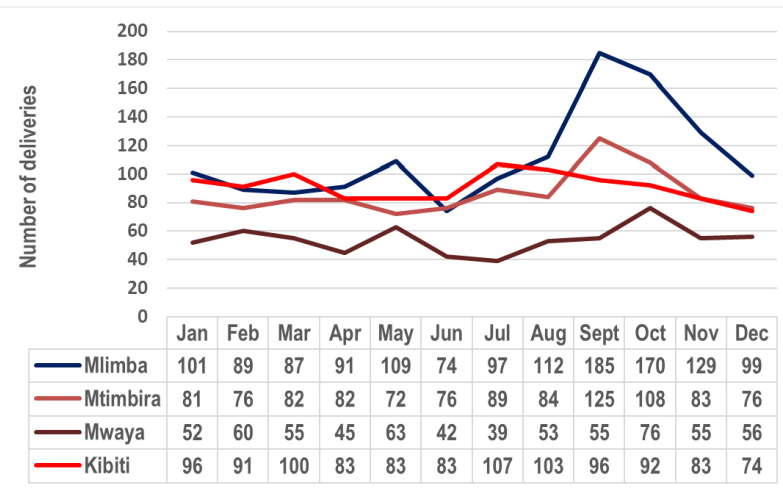

Figure 5. Trend in the monthly deliveries after project phase-out in 2013 in four remote health centers in Tanzania

\subsubsection{Continuity of $\mathrm{CEmOC}$ services and capacity to de- liver $\mathrm{CEMOC}$}

Most of the staffs who were trained for managing upgraded health centers during project implementation are still there. At Kibiti health centre the council has managed to increase number of health providers although the budget for equipments and other supplies is still the same as non-upgraded health facility. Unfortunately for Mlimba, Mtimbira and Mwaya health centres the number of health providers is still the same and the providers were complaining to be overworked although their distribution of drugs has been increased. There was additional critical services and benefits available at upgraded health centers, for example blood transfusion services were available; those women in need were safely transfused instead of being referred to district hospitals and resuscitation to newborns “... Laboratory services brought a lot of challenges, because many people from 
the two wards and all dispensaries around this area are coming here for laboratory services (tests).This services has increased the number of people here especially blood transfusion. This means in early days before the availability of laboratory services or upgrading the health centre, all patients were referred to Mahenge district Hospital, but this time all patients are handled here at our health facility" $\mathrm{HF}$ In charge-Mwaya upgraded health center.

\subsubsection{Sustainability challenges}

There has been notably increase in service utilisation particularly number of deliveries although the drugs and supplies like reagents and blood is still not enough. There has been a problem of electricity and facilities sometimes can not afford to pay for it. The interviewees reported that motivation in terms of allowance, staff houses, supervision money and fresher training is lacking. However, the upgrading also stimulated other initiatives such as plans for Kibiti upgraded health center and Mtimbira upgraded health center to be upgraded to a hospitals. A Pediatric ward for the Kibiti upgraded health center is under construction.

\section{Discussion}

In the past two decades, facility health related interventions have generated excitement, innovation, and exploration of alternative approaches. This article explores the tensions that health researchers face about transferring innovations to health systems, changing program delivery from intervention context controlled by researchers to program delivery controlled by health facilities and community organizations, as well as sustaining long term effects of interventions.

Essential goals of any facility and community based interventions include the long-term preservation of effects and fostering of collaboration between researchers, health facilities and local leaders. This paper reviews the challenges associated with transferring innovations to health systems, changing program delivery from an intervention context controlled by researchers to program delivery controlled by local organizations, and sustaining long-term effects of interventions. It is suggested that researchers who develop and implement health related interventions in diverse health areas need to confront several issues: (a) fostering effective long-term relationships between researchers and the community they study and in which they intervene and (b) designing and implementing interventions that are useful to community systems after the formal phase of research ends.

Lessons from the EMPOWER project show that upgrading health centers to provide emergency obstetric care is a possible endeavor. The results showed that utilization of health services can dramatically increase and eventually the entire system can be influenced. Therefore it was important to generate practical evidence and challenges which will enhance understanding on how to achieve sustainability on health interventions after grant period.

System bottlenecks on finance allocation to upgraded health centers is one of the issues. It has been noted that the upgraded health centers are getting the same amount of budget, drugs and other supplies like non-upgraded health centers. The study revealed that the upgraded health centers are still treated as the normal health centers by their respective district councils. As the result, services could not match with demand and utilization level, as it has been reported frequent stock out of drugs, equipment and supplies for all upgraded health centers.

With respect to other systemic challenges which includes leadership continuity, planning capacity and skills management findings are in line with what has been reported by Mona C. Shediac-Rizkallah and Lee R. Bone, 1998. ${ }^{[12]}$ Majority of developing countries likewise in our study changes do occur regularly in key leadership posts within the health system and you may find that within project time the leaders and health workers are changed several times, so it becomes difficult to retain the skills they get during project implementation.

It has been noted that most of challenges which have been associated with sustaining health intervention in one way or another they affect continuity of CEmOC services and the capacity to deliver CEmOC because the health workers who are key actors are limited to perform their job. For example in most cases the health workers are demotivated by being overworked yet they are not getting any allowance, they don't have staff houses, they don't receive regular supervision and feedback as well as refresher trainings.

Regional Health Management Teams and Zonal Training Centers/Resource Centers Regional are two mechanisms that have been proposed for strengthening local capacity both seen by the MOHSW as a means of ensuring the scaling up the project as well as ensuring the sustainability of introduced changes. Although the EMPOWER project did not engage directly with either of these, it also appeared that both these structures require significant capacity development if they are to provide the level of support needed for scaling up to the districts.

While we believe that the project should be able, within its implementation period, to test the effectiveness of the interventions being put in place, most of them are not designed to assess the effectiveness and potential for sustainability of these interventions or their replicability and scaling up to 
other parts. For the case of sustainability of $\mathrm{MNCH}$ related projects, a number of inter-related aspects of sustainability should be referred. These include continued ownership, provision of adequate resources, and retention of technical and managerial skills; the loss of any of these could endanger the continued levels of service access and quality.

It is important that the project itself together with key country stakeholders and donors to develop a strategy for the eventual wind-down of the project and hopefully, transfer key functions to other agencies. It is also considered that, whenever donor support for projects ends, there should be a continued need for such an implementation research capacity at different levels in the health system. This would provide ongoing support to decision-making within a constantly changing environment and, more specifically, support in the scale-up of lessons from the project. At the national level, the consideration should be given to the institutionalisation of a continued function of health system strengthening implementation research.

The quality assurance of the data and status of data collection in rural parts of developing countries have their limitations. ${ }^{[13]}$ Sometimes it is not possible to achieve a record of all $\mathrm{MNCH}$ indicators. Under-reporting of cases or incomplete recording of indicators remain a challenge and could have had an effect on our results. However, irrespective of the noted challenges, information and data collected remains crucial in understanding the dynamics happening in the district for planning and evaluation of the health system performance.

\section{Conflicts OF InTEREST Disclosure}

Authors declare that they have no competing interests.

\section{REFERENCES}

[1] Hill K, Thomas K, AbouZahr C, et al. Estimates of maternal mortality worldwide between 1990 and 2005: an assessment of available data. Lancet. 2007; 370(9595): 1311-1319. http://dx.doi.org /10.1016/S0140-6736(07)61572-4

[2] National Bureau of Statistics (NBS) (Tanzania), ICF Macro. Tanzania Demographic and Health Survey 2010. NBS and ICF Macro, Dar es Salaam. 2011.

[3] The United Republic of Tanzania Ministry of Health and Social Welfare. The National Road Map Strategic Plan to Accelerate Reduction of Maternal, Newborn and Child Deaths in Tanzania. April 2008; $1-76$.

[4] Mrisho M, Obrist B, Schellenberg JA, et al. The use of antenatal and postnatal care: perspectives and experiences of women and health care providers in rural southern Tanzania. BMC Pregnancy Childbirth. Mar 4 2009; 9: 10. http://dx . doi . 10.1186/1471-2393-9-10

[5] The United Republic of Tanzania Ministry of Health and Social Welfare. Circulation of Draft One of HSSP IV - All members0001.pdf. 2015.

[6] Altman DG. Challenges in Sustaining Public Health Interventions. Heal Educ Behav. Nov 2006; 36(1): 24-28. http://dx.doi.org /10.1177/1090198107299788
[7] Wiltsey Stirman S, Kimberly J, Cook N, et al. The sustainability of new programs and innovations: a review of the empirical literature and recommendations for future research. Implement Sci. Mar 14 2012; 7: 17.

[8] Batan M, Butterfoss F, Jaffe A, et al. Sustainability Planning. 2014; $1-34$.

[9] Altman DG. Sustaining interventions in community systems: on the relationship between researchers and communities. Health Psychol.1995; 14(6): 526-536. PMid: 8565927. http://dx.doi.org /10.1037/0278-6133.14.6.526

[10] Paxton A, Maine D, Hijab N. Using the UN Process Indicators of Emergency Obstetric Services. 2003.

[11] AMDD Working Group on Indicators. Using UN process indicators to assess needs in emergency obstetric services:Bhutan,Cameroon and Rajasthan,India. Int J Gynaecol Obstet. Jun 2002; 77(3): 277-84. PMid: 12065143.

[12] Shediac-rizkallah MC, Bone LR. Planning for the sustainability of community-based health programs: conceptual frameworks and future directions for research. Practice and Policy. 1998; 13(1): 87-108. http://dx.doi.org/10.1093/her/13.1.87

[13] Mwageni E, Momburi D, Juma Z, et al. Tanzania essential health interventions project. Adult morbidity and mortality. 1998. 